\title{
Luxação traumática atlanto-occipital: relato de caso*
}

\author{
Traumatic atlanto-occipital dislocation: a case report
}

\author{
Helton Defino ${ }^{1}$, Maximiliano Aguiar Porto², Carlos Fernando Pereira da Silva Herrero², \\ Carlos Frederico Wanderley Estelita Romeiro ${ }^{2}$, Marcello Henrique Nogueira Barbosa ${ }^{3}$
}

\section{RESUMO}

A luxação traumática atlanto-occipital é lesão rara, de incidência desconhecida e está associada a elevada taxa de mortalidade. Os autores relatam o diagnóstico, tratamento e seguimento de dois anos de uma paciente de 25 anos de idade, vítima de acidente automobilístico e luxação atlanto-occipital traumática confirmada por exames de imagem.

\section{Descritores - Articulação atlanto-occipital/lesões; Trauma- tismos da coluna vertebral/diagnóstico; Trau- matismos da coluna vertebral/cirurgia; Rela- to de casos [Tipo de publicação]}

\section{ABSTRACT}

Traumatic atlanto-occipital dislocation is a rare lesion whose incidence is not know, and which is

* Trabalho realizado no Hospital das Clínicas da Faculdade de Medicina de Ribeirão Preto da Universidade de São Paulo - Departamento de Biomecânica, Medicina e Reabilitação do Aparelho Locomotor - Ribeirão Preto (SP), Brasil.

1. Professor Titular do Departamento de Biomecânica, Medicina e Reabilitação do Aparelho Locomotor do Hospital das Clínicas da Faculdade de Medicina de Ribeirão Preto da Universidade de São Paulo - USP - Ribeirão Preto (SP), Brasil.

2. Residente do Departamento de Biomecânica, Medicina e Reabilitação do Aparelho Locomotor do Hospital das Clínicas da Faculdade de Medicina de Ribeirão Preto da Universidade de São Paulo - USP - Ribeirão Preto (SP), Brasil.

3. Professor da Divisão de Radiologia do Departamento de Clínica Médica da Faculdade de Medicina de Ribeirão Preto da Universidade de São Paulo - USP - Ribeirão Preto (SP), Brasil.

Endereço para correspondência: Helton L.A. Defino, Av. Bandeirantes, 3.900, 11ํo andar - 14048-900 - Ribeirão Preto, SP. Tel.: (16) 3602-2513. E-mail: hladefin@fmrp.usp.br

Recebido em 14/1/08. Aprovado para publicação em 4/3/08. Copyright RBO2008 associated to a high rate of mortality. The authors report the diagnosis, treatment, and two-year followup of a 25 year-old patient who had had an automobile accident with imaging-confirmed traumatic atlanto-occipital dislocation.

\section{Keywords - Atlanto-occipital joint/injuries; Spinal injuries/diagnosis; Spinal injuries/surgery; Case reports [Publication type]}

\section{INTRODUÇÃO}

O primeiro relato da luxação traumática atlanto-occipital (LTAO) ocorreu em 1908, no qual um marinheiro de 19 anos de idade sofreu acidente durante a prática de exercícios de ginástica ${ }^{(1)}$. O paciente, que apresentava quadriplegia completa e insuficiência respiratória, foi a óbito após ser submetido a laminectomia descompressiva.

A luxação traumática atlanto-occipital é lesão rara e sua incidência exata permanece desconhecida. Nas vítimas fatais de acidente automobilístico foi observada incidência de 20-25\% de LTAO, documentada por exames radiográficos ${ }^{(2)}$. Em nosso meio, não verificamos na literatura pertinente relatos desse tipo de lesão.

Os pacientes que sobrevivem a essa lesão apresentam quadro clínico variado que não se correlaciona com o tipo da lesão atlanto-occipital. Os sintomas incluem dor cervical associada à limitação da mobilidade, torcicolo, paralisia dos nervos cranianos, sinais e sintomas sistêmicos de isquemia da circulação vertebrobasilar, déficit neurológico e coma ${ }^{(3-4)}$. Os sobreviventes com ausência completa de lesão neurológica são $\operatorname{raros}^{(4)}$. 


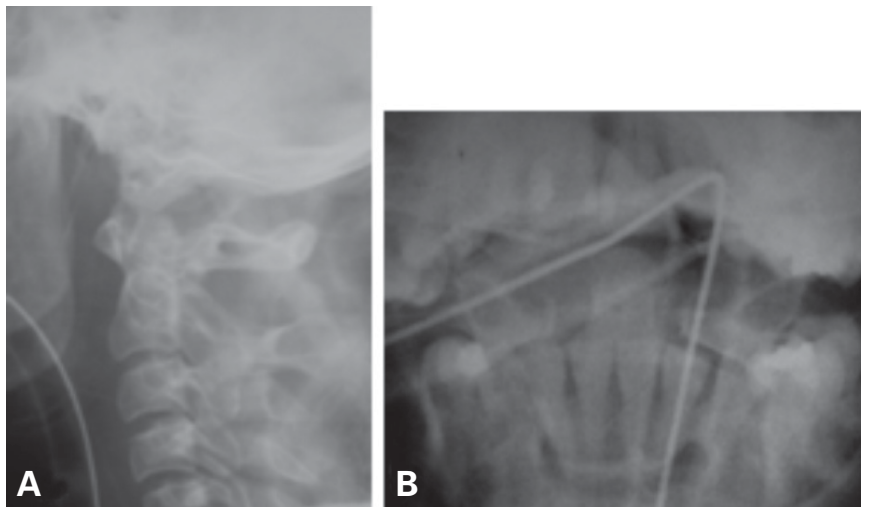

Figura 1 - Radiografias em perfil (A) e transoral (B) da coluna cervical evidenciando assimetria das relações anatômicas

A maior parte dos pacientes que sobreviveram à luxação atlanto-occipital traumática foi relatada na ultima década. Esse fato reflete a melhora do nível do atendimento de urgência, imobilização, transporte durante o atendimento inicial, novas técnicas de diagnóstico e maior índice de suspeição diagnóstica desse tipo de lesão(5-6).

Relatamos o tratamento e seguimento de uma paciente portadora de luxação atlanto-occipital traumática, que sobreviveu a esse tipo de lesão e apresentou recuperação do déficit neurológico durante seu seguimento.

\section{RELATO DO CASO}

Paciente do sexo feminino, 25 anos de idade, era a passageira dianteira de um veículo que foi atingido na sua parte lateral esquerda por um caminhão em alta velocidade. Após a colisão, o automóvel foi arrastado por cerca de 100 metros.

No momento da admissão hospitalar, a paciente apresentava o nível de consciência rebaixado, com 12 pontos pela escala de coma de Glasgow ${ }^{(7)}$, e queixava-se de dor abdominal, dor cervical e dispnéia. Apresentava aumento do volume abdominal, redução do murmúrio vesicular no hemitórax direito e sinais de instabilidade hemodinâmica. A avaliação neurológica ficou prejudicada pelo nível de consciência da paciente, mas foi observada discreta diminuição da força muscular (grau 4) no membro superior e inferior esquerdo. O hemotórax foi diagnosticado e a paciente foi também submetida à drenagem do tórax sob anestesia local na

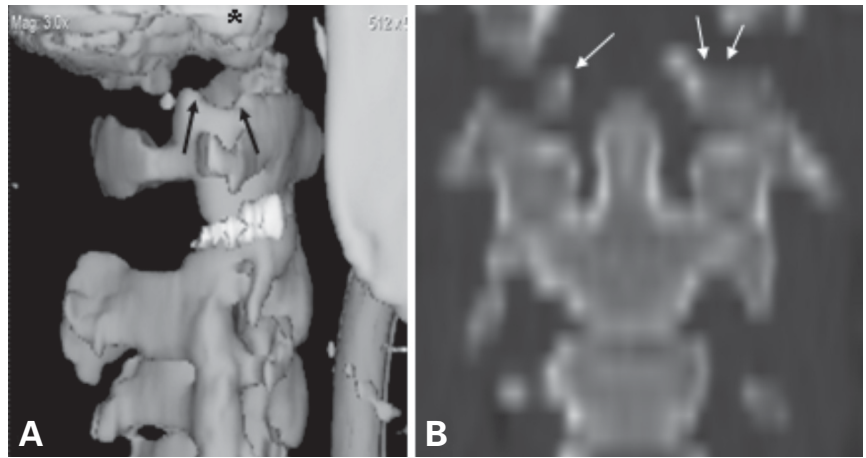

Figura 2 - Reconstrução sagital (A) e coronal (B) da tomografia computadorizada. Observar na reconstrução sagital (A) a luxação anterior do côndilo occipital (asterisco) sobre a superfície articular do áxis (setas pretas) e, na reconstrução coronal (B), a fratura bilateral do côndilo occipital (setas brancas).

sala de urgência, com a retirada de $1.150 \mathrm{ml}$ de sangue. A paciente foi submetida a laparotomia exploradora de urgência, tendo sido realizada esplenectomia devido à lesão irreparável do baço.

As radiografias em perfil e transoral da coluna cervical (figura 1) apresentavam alterações das relações anatômicas e suspeitas de lesão da coluna cervical alta, que foi confirmada por meio de tomografia computadorizada (TC). A tomografia computadorizada com reconstrução sagital e coronal evidenciou fratura bilateral dos côndilos occipitais, com luxação atlanto-occipital anterior (figura 2). As imagens da ressonância nuclear magnética (RNM) mostravam o aumento de sinal de líquido na articulação atlanto-occipital, lesão completa da membrana tectória, hematoma retroodontóide e hematoma anterior ao atlas (figura 3). A relação de Powers era de 0,78 e a distância entre o básio e o odontóide, de $0,7 \mathrm{~cm}$; estavam dentro dos limites dos valores considerados normais.

A paciente apresentou complicações clínicas no decorrer da sua internação (pneumonia, fungemia e distúrbios hidroeletrolíticos) e o tratamento cirúrgico pôde somente ser realizado 27 dias após o trauma. Durante esse período, a imobilização cervical foi realizada com o colar do tipo Philadelphia. O tratamento cirúrgico realizado foi a redução anatômica por meio do posicionamento da paciente na mesa cirúrgica com o auxilio de intensificador de imagens (figura 4) seguida de fixação occipitocervical posterior (occipito-C3) e artrodese com enxerto ósseo autólogo corticoesponjo- 


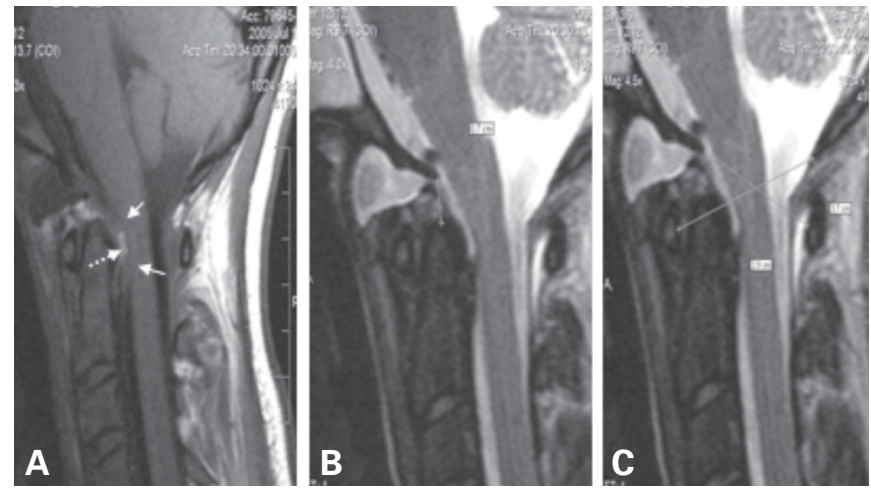

Figura 3 - Imagens da ressonância magnética. A) Imagem sagital ponderada em T1 evidenciando a ruptura da membrana tectória (setas preenchidas) e hematoma retroodontóide (seta tracejada). B) Imagem sagital ponderada em T2 mostrando a distância entre o básio e o odontóide $(0,7 \mathrm{~cm})$. C) Relação de Powers $\mathrm{BC} / \mathrm{AO}(0,78)$.
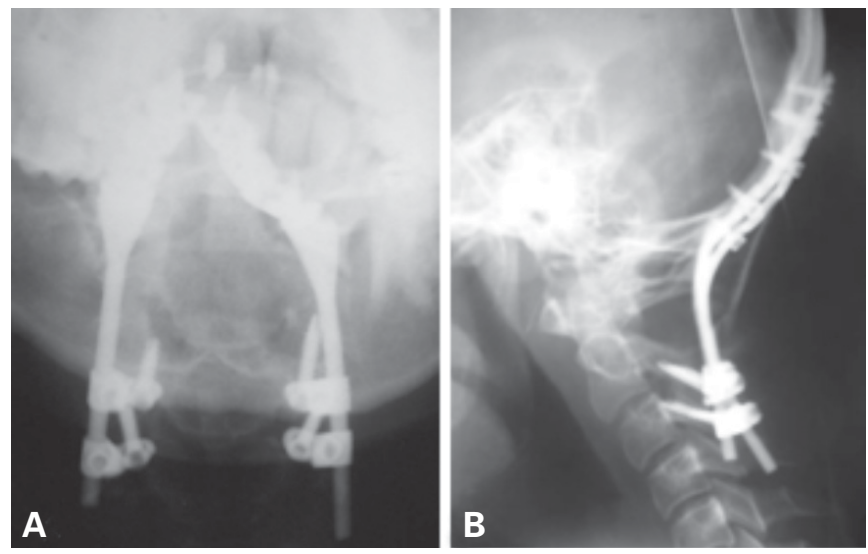

Figura 5 - Radiografia pós-operatória inicial na incidência ântero-posterior (A) e perfil (B) da artrodese occipito-C3

so do osso ilíaco (figura 5). O quadro neurológico não apresentou alteração no período pós-operatório e a paciente manteve força muscular de grau 4 no membro superior e inferior esquerdo.

A paciente apresentou retorno gradual da força muscular do membro superior e inferior esquerdo e, a partir do quarto mês de seguimento, demonstrava força muscular normal. A paciente engravidou com três meses de pós-operatório e não apresentou nenhuma alteração durante o período pré-natal ou pós-natal. Aos dois anos de seguimento, a artrodese occipitocervical apresentava sinais de consolidação, a posição inicial foi mantida (figura 6 ) e a paciente não apresentava limitação funcional.

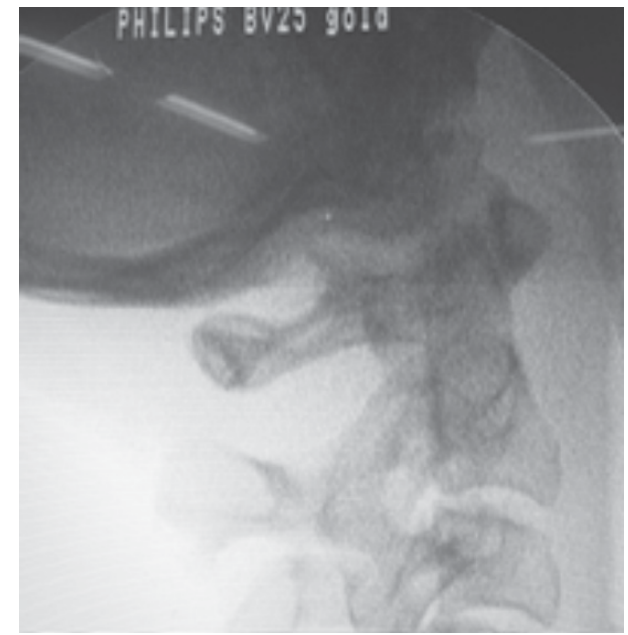

Figura 4

Imagem fluoroscópica intra-operatória em perfil da transição occipitocervical após a redução manual com o paciente posicionado na mesa cirúrgica

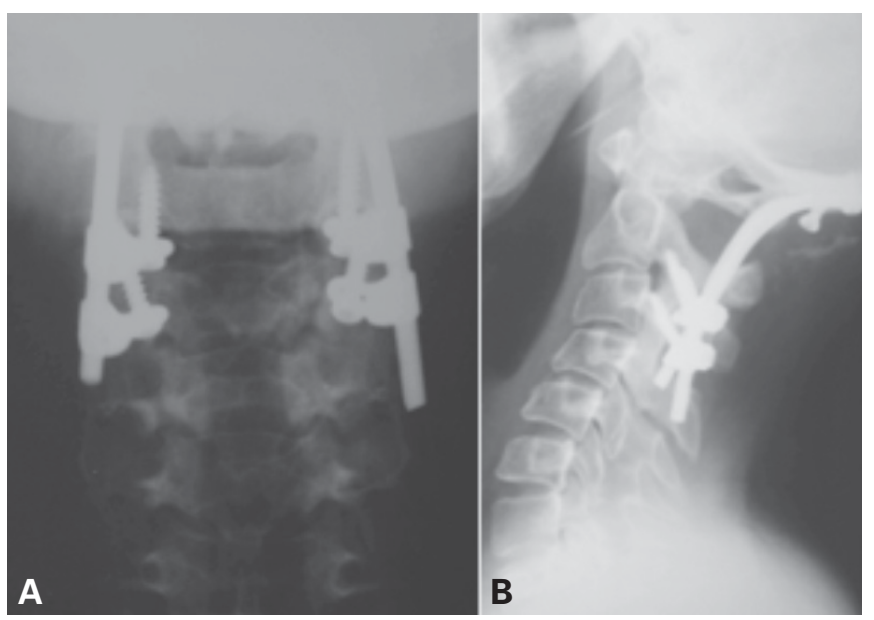

Figura 6 - Radiografias nas incidências ântero-posterior (A) e perfil (B) com dois anos de seguimento pós-operatório

\section{DISCUSSÃO}

A incidência e a prevalência da LTAO são desconhecidas devido às vítimas fatais com esse tipo de lesão. Estudos realizados durante autópsias indicam que a freqüência de LTAO pode ser relativamente alta. Alker et $a l^{(8)}$ observaram, entre 76 vítimas fatais de lesões cervicais relacionadas com acidente de trânsito, 19 casos de LTAO. Bucholz et al observaram 112 vítimas fatais de acidentes automobilísticos, nas quais LTAO foi a lesão isolada mais freqüentemente observada na coluna cervical dos politraumatizados, com incidência de $8 \%{ }^{(2)}$. Observaram também que a incidência dessa lesão foi maior nas crianças $(15 \%)$ que nos adultos (6\%). Nas crianças, a junção craniovertebral é menos 
estável, pois seus côndilos occipitais são relativamente menores e a junção craniovertebral é horizontal ${ }^{(9)}$. A maioria dos casos relatados de pacientes que sofreram LTAO ocorreu nas ultimas décadas ${ }^{(10)}$ e o aumento da sobrevida, provavelmente, é conseqüência da melhor qualidade do atendimento de urgência e melhora no diagnóstico dessas lesões ${ }^{(5-6)}$.

$\mathrm{O}$ mecanismo de lesão permanece controverso. Autores acreditam que a hiperextensão, causando a ruptura da membrana tectória, seja a causa principal ${ }^{(3,9,11)}$, enquanto outros consideram que o componente de flexão lateral também seja necessário(4,12) . A flexão occipitocervical é limitada pelo contato do processo odontóide com a porção anterior do forame magno, a hiperextensão limitada pela membrana tectória e a flexão lateral pelos ligamentos alares ${ }^{(13)}$. A secção dos ligamentos alares e da membrana tectória permite o deslocamento anterior do crânio com relação à coluna vertebral. É necessária a ruptura completa de todas as estruturas ligamentares entre o occipício e o complexo atlanto-axial para que ocorra a $\mathrm{LTAO}^{(14)}$. Os ligamentos alares estão mais distendidos e, conseqüentemente, mais vulneráveis à lesão, quando a cabeça está rodada e, adicionalmente, fletida ${ }^{(15)}$.

A manifestação clínica depende do nível e gravidade da lesão. Pode variar desde a parada cardiorrespiratória com quadriplegia a exame neurológico normal; algumas vezes, o paciente apresenta somente desconforto e rigidez cervical ${ }^{(16-17)}$. A avaliação neurológica do paciente com lesão da coluna cervical alta é, muitas vezes, difícil, devido à presença de traumatismo craniencefálico associado; a origem do déficit neurológico pode permanecer indefinida na avaliação inicial $^{(4,9,18-19)}$. Vários quadros neurológicos têm sido relatados e o mais frequiente é o déficit neurológico completo $^{(20)}$. As lesões medulares ocorrem devido ao trauma direto ou secundárias a hematoma extradural e se manifestam como hemiparesias, quadriparesias ou paresia bilateral dos membros superiores ${ }^{(6,21)}$. O mecanismo de tração axial, sofrida pela medula e nervos cranianos como conseqüência da separação entre os côndilos occipitais e o atlas, seria o responsável pela presença de lesões dos nervos cranianos, principalmente paralisia do sexto, nono e $12^{\circ}$ pares, freqüente nos pacientes com $\mathrm{LTAO}^{(9)}$. Foram relatadas lesões secundárias das artérias vertebrais, incluindo compressão, lesão da íntima ou trombose ${ }^{(22)}$. A lesão é instável e pode ocorrer piora do quadro neurológico quando o diagnóstico não é realizado na fase aguda ${ }^{(9,19,21,23)}$.

O diagnóstico de luxação traumática atlanto-occipital (LTAO) é freqüentemente realizado com base na combinação dos sinais e sintomas clínicos e nas radiografias em perfil da coluna cervical. Essa lesão deve sempre ser suspeitada nos pacientes com distúrbios cardiorrespiratórios e alterações neurológicas e que foram vítimas de acidentes; muitas vezes, assim como ocorreu com a nossa paciente, o diagnóstico inicial pode não ser feito ${ }^{(17,20)}$. O diagnóstico de LTAO por meio das radiografias simples apresenta baixa sensibilidade e especificidade ${ }^{(24)}$. É necessária técnica adequada para a realização do exame, assim como posicionamento em perfil verdadeiro, para a identificação confiável das estruturas ósseas utilizadas na avaliação. Foram descritas relações entre as referências anatômicas da transição occipitocervical para o diagnóstico das lesões desse segmento vertebral. A linha de Wackenheim estende-se distalmente ao longo da superfície posterior do clivo ${ }^{(14)}$. Essa linha deveria tangenciar a ponta do odontóide. Se o occipício está deslocado anteriormente, a linha irá intersectar o odontóide e, se o occipício sofreu distração ou deslocamento posterior, a linha estará afastada do odontóide. A relação de Powers avalia a razão entre duas linhas, a distância entre o básio (B) e o arco posterior do atlas (C) e a distância entre o opístio (O) e o arco anterior do atlas (A) ${ }^{(21)}$. Em indivíduos normais, a média de BC/AO é de 0,77 . Um valor maior do que 1 é indicador de luxação anterior. Dublin et al sugeriram utilizar a distância entre a cortical mandibular posterior e o arco anterior de $\mathrm{C} 1$ e a distância entre a cortical mandibular posterior e o odontóide para avaliar o alinhamento atlanto-occipital ${ }^{(3)}$. Situações como fratura da mandíbula, flexão e extensão cervical ou, até mesmo, certo grau de rotação tornam essa técnica freqüentemente não reprodutível ${ }^{(12)}$. Nos exames de imagem de nossa paciente, a relação de Powers era de 0,78 e a distância entre o básio e o odontóide, de $0,7 \mathrm{~cm}$; estavam dentro dos limites dos valores considerados normais. Autores consideram a 
linha de Wackenheim e a distância básio-odontóide as medidas mais sensíveis ${ }^{(23-24)}$, enquanto outros evidenciaram maior sensibilidade utilizando a relação de Powers $^{(12,25)}$. Esses métodos de avaliação não são apropriados se houver fratura do atlas ou do áxis ou presença de má formação óssea associada ${ }^{(12)}$. Um método confiável de avaliar a presença de LTAO, quando o diagnóstico não puder ser realizado por meio de radiografias simples em perfil da coluna cervical, é a tomografia computadorizada, a qual fornece melhor visualização da articulação atlanto-occipital ${ }^{(19,23)}$; é considerada por muitos autores como o melhor método diagnóstico da LTAO e que, em nossa paciente, colaborou para a realização do diagnóstico ${ }^{(19,26)}$. A ressonância magnética pode mostrar também avulsões ligamentares, mas é mais útil para diagnosticar a presença de hematomas associados, lesões de tecidos moles e a integridade dos elementos neurais ${ }^{(27-28)}$, tendo sido esse feito confirmado em nossa paciente.

Traynelis et al sugeriram a presença de três tipos específicos de LTAO: o tipo I envolve o deslocamento anterior do occipício com relação ao atlas; o tipo II é uma distração longitudinal com separação do occipício e do atlas; e o tipo III apresenta o deslocamento posterior do occipício sobre o atlas ${ }^{(6)}$.

O tratamento na fase aguda tem como principal objetivo a manutenção das funções vitais e a imobilização da coluna vertebral. A redução e o realinhamento da luxação podem causar lesões acidentais e devem ser cuidadosamente realizadas e sob exame radiológico ou fluoroscópico. Apesar de toda controvérsia envolvendo a utilização da tração esquelética cervical como meio de redução da lesão, existe concordância por parte dos cirurgiões de que o melhor meio de imobilização provisória da LTAO seria o halo-colete e não o colar do tipo Philadelphia, como foi utilizado em nossa paciente ${ }^{(5,10,29)}$. No entanto, nem sempre o estado geral do paciente e as lesões associadas permitem esse tipo de imobilização na fase aguda. A utilização de tração axial com leve peso para o realinhamento das luxações tem sido preconizada ${ }^{(6,9,28)}$. No entanto, a distração progressiva do occipício e do atlas tem sido relatada com esta manobra ${ }^{(3)}$. A tração cervical e o colar cervical como forma de estabilização da luxação têm sido contra-indicados por autores ${ }^{(4,30)}$, que preconizam a realização do tratamento cirúrgico de urgência nos pacientes sem déficit neurológico ou com perda parcial da função neurológica ${ }^{(27)}$. A artrodese occipitocervical é o método de escolha para o tratamento da LTAO. A lesão é predominantemente ligamentar e o tratamento conservador não restabelece a estabilidade necessária para a função fisiológica.

O tratamento cirúrgico da LTAO reflete a influência do desenvolvimento das fixações vertebrais. De início, a artrodese era realizada com enxerto ósseo e fixação com fios metálicos ${ }^{(18,31)}$, havendo necessidade da estabilização por meio de halo craniano durante várias semanas no pós-operatório, até a consolidação da artrodese. Atualmente, a utilização de sistemas mais rígidos de fixação do occipício às vértebras cervicais permite a mobilização e reabilitação precoce dos pacientes sem necessidade de imobilização externa como o halo gesso ${ }^{(32)}$.

Os eventos relacionados à LTAO da nossa paciente corroboram e ilustram as características dessa lesão, como a dificuldade do seu diagnóstico inicial; comprovam ainda a importância e validade do tratamento de emergência adequado. $\mathrm{O}$ tratamento adequado na fase aguda para a manutenção das funções vitais permite a realização do tratamento definitivo que pode conduzir à reabilitação do paciente e retorno às suas atividades normais após a ocorrência de uma lesão de alta gravidade e que pode evoluir para óbito ou lesão neurológica irreversível.

\section{REFERÊNCIAS}

1. Blackwood NJ. III. Atlo-occipital dislocation: a case of fracture of the atlas and axis, and forward dislocation of the occiput on the spinal column, life being maintained for thirty-four hours and forty minutes by artificial respiration, during which a laminectomy was performed upon the third cervical vertebra. Ann Surg. 1908;47(5):654-8.

2. Bucholz RW, Burkhead WZ. The pathological anatomy of fatal atlanto-occipital dislocations. J Bone Joint Surg Am. 1979; 61(2):248-50.

3. Dublin AB, Marks WM, Weinstock D, Newton TH. Traumatic dislocation of the atlanto-occipital articulation (AOA) with short-term survival. With a radiographic method of measuring the AOA. J Neurosurg. 1980;52(4):541-6. 
4. Gabrielsen TO, Maxwell JA. Traumatic atlanto-occipital dislocation; with case report of a patient who survived. Am J Roentgenol Radium Ther Nucl Med. 1966;97(3):624-9.

5. Levine AM, Edwards CC. Traumatic lesions of the occipitoatlantoaxial complex. Clin Orthop Relat Res. 1989; (239):53-68.

6. Traynelis VC, Marano GD, Dunker RO, Kaufman HH. Traumatic atlanto-occipital dislocation. Case report. J Neurosurg. 1986; 65(6):863-70. Erratum in: J Neurosurg. 1987;66(5):789.

7. Teasdale G, Jennett B. Assessment of coma and impaired consciousness. A practical scale. Lancet. 1974;2(7872):81-4.

8. Alker GJ Jr, Oh YS, Leslie EV. High cervical spine and craniocervical junction injuries in fatal traffic accidents: a radiological study. Orthop Clin North Am. 1978;9(4):1003-10.

9. Fruin AH, Pirotte TP. Traumatic atlantooccipital dislocation. Case report. J Neurosurg. 1977;46(5):663-6.

10. Przybylski GJ, Clyde BL, Fitz CR. Craniocervical junction subarachnoid hemorrhage associated with atlanto-occipital dislocation. Spine. 1996;21(15):1761-8.

11. Page CP, Story JL, Wissinger JP, Branch CL. Traumatic atlantooccipital dislocation. Case report. J Neurosurg. 1973; 39(3):394-7.

12. Pang D, Wilberger JE Jr. Traumatic atlanto-occipital dislocation with survival: case report and review. Neurosurgery. 1980;7(5):503-8.

13. Werne S. Studies in spontaneous atlas dislocation. Acta Orthop Scand Suppl. 1957;23:1-150.

14. Evarts CM. Traumatic occipito-atlantal dislocation. J Bone Joint Surg Am. 1970;52(8):1653-60.

15. Dvorak J, Panjabi MM. Functional anatomy of the alar ligaments. Spine. 1987;12(2):183-9.

16. Ide C, Nisolle JF, Misson N, Trigaux JP, Gustin T, De Coene B, et al. Unusual occipitoatlantal fracture dissociation with no neurological impairment. Case report. J Neurosurg. 1998; 88(4):773-6.

17. Jevtich V. Traumatic lateral atlanto-occipital dislocation with spontaneous bony fusion. A case report. Spine. 1989;14(1): 123-4.

18. Belzberg AJ, Tranmer BI. Stabilization of traumatic atlantooccipital dislocation. Case report. J Neurosurg. 1991;75(3): 478-82.
19. Gerlock AJ Jr, Mirfakhraee M, Benzel EC. Computed tomography of traumatic atlantooccipital dislocation. Neurosurgery. 1983;13(3):316-9.

20. DiBenedetto T, Lee CK. Traumatic atlanto-occipital instability. A case report with follow-up and a new diagnostic technique. Spine. 1990;15(6):595-7.

21. Powers B, Miller MD, Kramer RS, Martinez S, Gehweiler JA Jr. Traumatic anterior atlanto-occipital dislocation. Neurosurgery. 1979;4(1):12-7.

22. Lee C, Woodring JH, Walsh JW. Carotid and vertebral artery injury in survivors of atlanto-occipital dislocation: case reports and literature review. J Trauma. 1991;31(3):401-7.

23. Lee C, Woodring JH, Goldstein SJ, Daniel TL, Young AB, Tibbs PA. Evaluation of traumatic atlantooccipital dislocations. AJNR Am J Neuroradiol. 1987;8(1):19-26.

24. Bulas DI, Fitz CR, Johnson DL. Traumatic atlanto-occipital dislocation in children. Radiology. 1993;188(1):155-8.

25. Collalto PM, DeMuth WW, Schwentker EP, Boal DK. Traumatic atlanto-occipital dislocation. Case report. J Bone Joint Surg Am. 1986;68(7):1106-9.

26. Watridge CB, Orrison WW, Arnold H, Woods GA. Lateral atlantooccipital dislocation: case report. Neurosurgery. 1985; 17(2):345-7.

27. Guigui P, Milaire M, Morvan G, Lassale B, Deburge A. Traumatic atlantooccipital dislocation with survival: case report and review of the literature. Eur Spine J. 1995;4(4):2427.

28. Scott EW, Haid RW Jr, Peace D. Type I fractures of the odontoid process: implications for atlanto-occipital instability. Case report. J Neurosurg. 1990;72(3):488-92. Comment in: J Neurosurg. 1990;73(4):644.

29. Matava MJ, Whitesides TE Jr, Davis PC. Traumatic atlantooccipital dislocation with survival. Serial computerized tomography as an aid to diagnosis and reduction: a report of three cases. Spine. 1993;18(13):1897-903.

30. Rockswold GL, Seljeskog EL. Traumatic atlantocranial dislocation with survival. Minn Med. 1979;62(3):151-2, 154.

31. Eismont FJ, Bohlman HH. Posterior atlanto-occipital dislocation with fractures of the atlas and odontoid process. J Bone Joint Surg Am. 1978;60(3):397-9.

32. Papadopoulos SM, Dickman CA, Sonntag VK, Rekate HL, Spetzler RF. Traumatic atlantooccipital dislocation with survival. Neurosurgery. 1991;28(4):574-9. 